\title{
Carbon Dioxide Sequestration Through Hyper- Alkalination of the Liquid- Condensed Phase
}

\author{
MARK A. Bewernitz, ${ }^{1 *}$ MATthew Ginder-Vogel, ${ }^{3}$ \\ JACOB SCHNEIDER, ${ }^{1}$ CHRISTOPHER L. CAMIRÉ, ${ }^{1}$ SEUNG- \\ HeE Kang, ${ }^{1}$ William L. Bourcier, ${ }^{1}$ Richard Wade, ${ }^{1}$ \\ BRENT R. CONSTANTZ ${ }^{1,2} *_{\text {12 }}$
}

We provide evidence that the bicarbonate-rich liquid condensed phase (LCP) does not require supersaturated conditions to form and is neither mineral specific, nor transient but rather is a fundamental electrolyte behavior exhibited by the bicarbonate ion which exists at undersaturated, and apparent equilibrium conditions. Stable LCP droplets at undersaturated conditions allow for a previously unknown of means to manipulate and store inorganic carbon in the solution state and the engineering of synthetic minerals with superior properties. Solutions containing bicarbonate ions such as seawater, natural brines, bovine serum and other mineralizing milieus have been discovered to contain hyperalkaline droplets of a separate, liquid condensed phase (LCP). The existence and unique composition of the LCP droplets has been confirmed by methods of Nanoparticle Tracking Analysis (NTA), Nuclear Magnetic Resonance (NMR), Fourier Transform Infrared (FTIR), Dissolved Inorganic Carbon analysis (DIC) and refractive index (RI) measurements. Carbon dioxide can be dissolved in solution through an aqueous reaction to form LCP. Precipitation of calcium carbonate by reaction with $\mathrm{Ca} 2+$ and LCP droplets appears to be a ubiquitous embodiment of carbonate mineral formation, including biomineralization. Reported herein are droplets of a liquid condensed phase (LCP) that are bicarbonate-rich, or hyperalkaline, relative to the bulk solution, in modern and ancient seawater compositions blood serum, electrolyte solutions forming calcium carbonate polymorphs similar to those seen in the mineralizing milieu in calcium carbonate skeletal biomineralization, and industrial carbon dioxide capture and mineralization solutions for carbon dioxide sequestration. LCP is present in simple sodium carbonate solutions over a range of $\mathrm{pH}$ as well as more complex solutions from which carbonate minerals are forming. This second phase requires that seawater, blood, the biomineralizing milieu, and many capture fluids in carbon dioxide sequestration be viewed as a two-phase system, rather than a one-phase system. As a result, calculated estimates of the parameters of these systems from two measured values do not fully constrain the system. When calcium is present in these systems, even at near-neutral $\mathrm{pH}$ where bicarbonate is dominant, the bicarbonate is particularly concentrated in the hyperalkaline LCP phase, which leads to calcium carbonate precipitation and evolution of carbon dioxide gas. 